\title{
Paeoniflorin Alleviates Abnormalities in Rats with Functional Dyspepsia by Stimulating the Release of Acetylcholine
}

This article was published in the following Dove Press journal:

Drug Design, Development and Therapy

\author{
Xuan Zou ${ }^{1,2, *}$ \\ Yang Wang ${ }^{1,2, *}$ \\ Yuheng Wang ${ }^{3}$ \\ Junting Yang ${ }^{3}$ \\ Huishu Guo ${ }^{1,2}$ \\ Zhengxu Cai iD ${ }^{3}$ \\ 'Central Laboratory, The First Affiliated \\ Hospital of Dalian Medical University, \\ Dalian I I600I, People's Republic of \\ China; ${ }^{2}$ Institute (College) Integrative \\ Medicine, Dalian Medical University, \\ Dalian I I6044, People's Republic of \\ China; ${ }^{3}$ Department of Neurology, The \\ First Affiliated Hospital of Dalian Medical \\ University, Dalian II600I, People's \\ Republic of China
}

*These authors contributed equally to this work

\begin{abstract}
Introduction: Paeoniflorin is a main active component in traditional Chinese medicine. Paeoniae alba radix is widely used as a spasmolytic and pain-relieving agent for abdominal spasmodic pain. Functional dyspepsia (FD) is characterized by pain or burning in the epigastrium, fullness, bloating and nausea. However, limited information is available about the effect of paeoniflorin on FD.
\end{abstract}

Materials and Methods: In this study, iodoacetamide or clonidine-induced FD rat models were established to investigate the impacts of paeoniflorin on FD induced by different pathophysiologic disturbances.

Results: We found the therapeutic effect of paeoniflorin through assessing the gastric emptying, gastric accommodation and visceral hypersensitivity. This function of paeoniflorin was related to the release of acetylcholine (ACh), which was accompanied by reduced acetylcholinesterase (AchE) activity in stomach and hypothalamus. Paeoniflorin administration inhibited the cyclo-oxygenase-2 (COX-2) expression and increased the level of ghrelin in the stomach. Besides, the levels of occludin and ZO-1 were elevated in the duodenum from paeoniflorin-treated rats, suggesting the impaired duodenal barrier was ameliorated.

Discussion: These results indicate that paeoniflorin possesses the ability to alleviate functional dyspepsia.

Keywords: paeoniflorin, functional dyspepsia, acetylcholine, gastric function

\section{Introduction}

Functional dyspepsia (FD) is a highly prevalent gastrointestinal disorder characterized by a sensation of pain or burning in the epigastrium, fullness during or after meal, bloating, and nausea. ${ }^{1}$ The global prevalence of FD is about $20 \%{ }^{2}$ At present, therapies for FD, including pharmacotherapy, are not yet been fully effective. ${ }^{3}$ This condition adversely affects patients' quality of life and working efficiency. ${ }^{4}$ Consequently, the identification of efficacious treatments (such as natural products) that do not involve prescription drugs may be beneficial. According to Rome Consensus definition, FD is categorized into postprandial distress syndrome (PDS, characterized by postprandial fullness and early satiation) and epigastric pain syndrome (EPS, characterized by epigastric pain and burning). ${ }^{5}$ The above adverse symptoms can be caused by delayed gastric emptying (about $20-50 \%$ of patients with FD), impaired gastric accommodation to a meal (about $40 \%$ of patients with FD) and visceral hypersensitivity to gastric distension (34\% of patients with FD). ${ }^{6}$
Correspondence: Zhengxu Cai Department of Neurology, The First Affiliated Hospital of Dalian Medical University, 222 Zhongshan Road, Dalian I I600I, People's Republic of China Email caizhengxu999@I26.com

Huishu Guo

Central Laboratory, The First Affiliated

Hospital of Dalian Medical University, 222

Zhongshan Road, Dalian II600I, People's

Republic of China

Tel +86-4l I-83635963 ext 7255

Email guohuishul@I26.com 
Acetylcholine (ACh), an excitatory neurotransmitter, promotes contraction and peristalsis of gastrointestinal smooth muscle. Dopamine D2 receptors and 5-hydroxytryptamine $\left(5-\mathrm{HT}_{4}\right)$ receptors affect the release of $\mathrm{ACh}$ and thereby resulting in gastrointestinal motility disorder. Dopamine D2 receptor antagonists and $5-\mathrm{HT}_{4}$ receptor agonists as prokinetic drugs are extensively used in the therapy of FD, because they can increase gastric contractility. $^{7,8}$ Metoclopramide, domperidone, and itopride hydrochloride (itopride) are dopamine D2 receptor antagonists, while mosapride citrate (mosapride), cisapride monohydrate (cisapride), and tegaserod are $5-\mathrm{HT}_{4}$ receptor agonists. However, the therapeutic effects of these drugs are unsatisfactory in FD patients. ${ }^{9}$ Previous researches have confirmed that metoclopramide causes severe neurological adverse effects, ${ }^{10}$ and domperidone facilitate occurrence of arrhythmias. ${ }^{11}$ Cisapride contributed to the release of $\mathrm{ACh}$ from myenteric neurons via $5-\mathrm{HT}_{4}$ receptor agonism, but was abandoned for QT interval prolongation and fatal arrhythmias. ${ }^{12}$ In the past few years, tegaserod was used to treat the irritable bowel syndrome with constipation, but it had been withdrawn due to an increased incidence of cardiovascular ischemic events. ${ }^{12}$ Itopride and mosapride were efficacious in the treatment of FD, but the use of these drugs was clinically limited because of underlying adverse effects. ${ }^{2,13}$ Thus, safer therapies for FD, such as employing natural products, should be developed.

Paeoniae alba radix (also named White Paeony Root or Baishao in Chinese) is one of the Chinese traditional tonic crude drugs, which is the dried peeled root of Paeonia lactiflora Pall (family Paeoniaceae). It is widely used as a spasmolytic and pain-relieving agent to treat menstrual disorders and to relieve abdominal spasmodic pain. ${ }^{14}$ Paeoniflorin is a monoterpene glycoside isolated from the aqueous extract of the dry root of P. lactiflora Pall and is identified as a main active component in Paeoniae alba radix. Paeoniflorin had been found to exert many pharmacological effects, including an increase of cognitive ability, ${ }^{15}$ a reduction of MPTP-induced toxicity, ${ }^{16}$ and antidepressant effect. ${ }^{17}$ Recent studies suggested that paeoniflorin down-regulated the activity of acetylcholinesterase (AchE) in the hippocampus of rats, ${ }^{18}$ and up-regulated the expression level of 5-HT. ${ }^{19}$ Thus, we postulate that paeoniflorin may be a novel natural drug for the treatment of FD. However, the gastroprokinetic and gastric emptying activities of paeoniflorin, including its underlying mechanisms, have yet to be fully elusive.
Gastric irritation with iodoacetamide (IA) in the neonatal period induces gastric hypersensitivity, motor dysfunction and damaged accommodation in adults. ${ }^{20}$ Neonatal gastric irritation has been regarded as a typical approach to establish a FD animal model. It has been known that clonidine (CD) as a $\alpha 2$-adrenoceptor agonist can inhibit the release of ACh from cholinergic neurons and thereby lead to gastric motility dysfunction. ${ }^{21}$ In this study, IA or CD-induced FD rat models were established to assess the impacts of paeoniflorin on FD induced by different pathophysiologic disturbances. The effects of paeoniflorin on $\mathrm{ACh}$, ghrelin, $\mathrm{COX}-2$ and adhesion proteins were further investigated.

\section{Materials and Methods Medicinal Materials}

Paeoniflorin, the aqueous extract of the dry root of P. lactiflora Pall, was obtained from Meilun Biotechnology Co., Ltd. (MB2113, Dalian, China).

\section{Animals}

Male Sprague-Dawley (SD) rats aged at 10-day old and 8 -week old were purchased from Liaoning HFK BIOSCIENCE Co., Ltd. (Beijing, China). All rats were housed under the following environmental conditions: 22 $\pm 1^{\circ} \mathrm{C}$ temperature, $45-55 \%$ humidity, $12 \mathrm{~h}$ light/dark cycle and freely access to food and water. All the procedures in animal experiments followed the guide for the care and use of laboratory animals and this study was approved by The First Affiliated Hospital of Dalian Medical University.

\section{Neonatal Gastric Irritation Animal Model}

Gastric hypersensitivity and gastric motor dysfunction in FD can be modeled in rat pups by gastric irritation as previously reported. ${ }^{22}$ Ten-day-old rat pups were randomly divided into the following group:

- Sham group (sucrose + normal saline; $\mathrm{n}=6$ );

- Control group (IA + normal saline; $\mathrm{n}=6$ );

- IA+paeoniflorin (15) group (IA $+15 \mathrm{mg} / \mathrm{kg}$ paeoniflorin; $\mathrm{n}=6$ );

- IA+paeoniflorin (30) group (IA $+30 \mathrm{mg} / \mathrm{kg}$ paeoniflorin; $n=6$ ).

Ten-day-old rat pups were given orally $0.2 \mathrm{~mL}$ of $0.1 \%$ IA (144-48-9, Aladdin, Shanghai, China; dissolved in $2 \%$ sucrose) for 6 consecutive days; sham pups received 
$0.2 \mathrm{~mL}$ of $2 \%$ sucrose alone. Subsequently, rat pups were returned to their cage. After 6 weeks, rats were intraperitoneally injected 15 or $30 \mathrm{mg} / \mathrm{kg}$ paeoniflorin for 2 consecutive weeks. Gastric function was detected $1 \mathrm{~h}$ after the last injection of paeoniflorin.

\section{CD-Induced Motility Dysfunction Model}

CD-induced FD rat model was established using the method of Kawachi et al. ${ }^{23}$ Eight-week old rats were randomly divided into the following group:

- Sham group (normal saline + normal saline; $\mathrm{n}=6$ );

- Control group (CD + normal saline; $n=6$ );

- $\mathrm{CD}+$ paeoniflorin (15) group $(\mathrm{CD}+15 \mathrm{mg} / \mathrm{kg}$ paeoniflorin; $\mathrm{n}=6$ );

- $\mathrm{CD}+$ paeoniflorin (30) group $(\mathrm{CD}+30 \mathrm{mg} / \mathrm{kg}$ paeoniflorin; $\mathrm{n}=6$ ).

Eight-week-old rats were intraperitoneally injected 15 or $30 \mathrm{mg} / \mathrm{kg}$ paeoniflorin for 2 consecutive weeks. Sham rats received normal saline alone. At $30 \mathrm{~min}$ after the last injection of paeoniflorin, CD at a dose of $100 \mu \mathrm{g} / \mathrm{kg}(20$,283-92-5, Sigma, St. Louis, Missouri, USA) or normal saline was subcutaneously administered. Gastric function was detected $30 \mathrm{~min}$ after $\mathrm{CD}$ treatment.

\section{Measurement of Gastric Emptying}

Gastric emptying in rats was detected with the phenol red method as previously described. ${ }^{21}$ Rats were fasted for 18 $\mathrm{h}$ and free access to water. Rats were then administered intragastrically $1.5 \%$ carboxymethylcellulose sodium salt supplemented with $0.05 \%$ phenol red $(0.5 \mathrm{~mL} / \mathrm{rat})$. Twenty minutes later, rats were euthanized and stomach was collected. After treatment with $10 \mathrm{~mL}$ of $0.1 \mathrm{M} \mathrm{NaHCO}_{3}$, gastric content harvested and centrifuged at $8000 \mathrm{~g}$ for $15 \mathrm{~min}$ (H1650-W, Xiangyi, Changsha, China). The amount of phenol red in the supernatant was measured at the absorbance at $558 \mathrm{~nm}$ using a microplate reader (ELX-800, BioTek, Vermont, USA). For the IA-FD model, phenol red was intragastrically administered to rats $30 \mathrm{~min}$ after the last injection of paeoniflorin. For the CD-FD model, rats were intragastrically administered phenol red at $5 \mathrm{~min}$ before the administration of clonidine.

\section{Gastric Accommodation and Gastric Sensitivity Tests}

Gastric accommodation and gastric sensitivity were detected using barostat and electrophysiological recorder as described previously. ${ }^{24,25}$ Rats were anesthetized and then a balloon with a maximum volume of $20 \mathrm{~mL}$ was connected to a polyethylene tube. The tube was introduced from the mouth to the stomach. A pair of electrodes was fixed into the trapezius muscle. After rats regained consciousness, the tubes were connected to a barostat (C\&J Technology, Toronto, Canada) and electrophysiological recorder (AD instrument, New South Wales, Australia). Rats receipted a series of $30 \mathrm{~s}$ balloon distensions: 20, 40, 60 and $80 \mathrm{mmHg}$ at an interval of $3 \mathrm{~min}$ between distensions. The volume of the balloon and electromyographic (EMG) was recorded. Gastric accommodation was calculated based on the changes in balloon volume $(\mathrm{mL})$ per $\mathrm{mmHg}$ during the distension, while gastric sensitivity is defined as the rate of change root mean square of EMG.

\section{AchE Activity Assay}

AchE activity assay was performed as previously reported. ${ }^{23}$ The stomach was harvested from fasted rats and mixed with a 10 -fold volume of $0.1 \mathrm{mM}$ sodium phosphate buffer ( $\mathrm{pH}$ 6.9) containing $10 \mathrm{mM}$ EDTA. The mixture was centrifuged for $1 \mathrm{~h}$ and sediment was washed twice with buffer. After treatment with buffer containing $1 \%$ Triton X-100 at $4^{\circ} \mathrm{C}$ for $1 \mathrm{~h}$, supernatant was collected by centrifugation $\left(8000 \mathrm{~g}, 4^{\circ} \mathrm{C}, 10 \mathrm{~min}\right)$ and was regarded as a stomach-derived fraction with AChE. Protein concentration was detected using a BCA Assay Kit (PC0020, Solarbio, Beijing, China) according to the manufacturer's instructions. AChE activity was determined using an Assay Kit (BC2020, Solarbio) according to the manufacturer's protocol. The absorbance at $412 \mathrm{~nm}$ was detected using a spectrophotometer (UV752N, Youke, Shanghai, China). Inhibition rate (\%) was calculated as the ratio of ACh activity in the presence of a test compound and sham activity in the absence of the test compound.

\section{Western Blot}

The duodenum was lysed on ice for $5 \mathrm{~min}$ in cell lysis buffer (P0013, Beyotime, Shanghai, China) containing 1 mM PMSF (ST506, Beyotime). Supernatants were centrifugation at $10,000 \mathrm{~g}$ for $5 \mathrm{~min}$ at $4{ }^{\circ} \mathrm{C}$ and collected. The protein concentration was detected by the BCA Assay Kit (P0011, Solarbio). Equal number of supernatants $(20 \mu \mathrm{L} /$ lane) was separated on sodium dodecyl sulfatepolyacrylamide gel and transferred into PVDF membrane (IPVH00010, Millipore, Billerica, USA). After blocking in $5 \%(\mathrm{~m} / \mathrm{v})$ skim milk (Q/NYLB 0039S, Yili, Huhehaote, China), membrane was incubated overnight at $4^{\circ} \mathrm{C}$ with 
the following primary antibodies: E-cadherin (1:500, 20,874-1-AP, Proteintech, Beijing, China), occludin (1:1000, 27,260-1-AP, Proteintech), zona occludens-1 (ZO-1, 1:1000, 21,773-1-AP, Proteintech), and $\beta$-actin (1:1000, sc-47,778, Santa Cruz, California, USA). After four 5-min washing with TBST buffer, membrane was incubated with HRP-conjugated goat anti-rabbit or antimouse (both 1:5000, A0208/A0216, Beyotime) secondary antibody for $45 \mathrm{~min}$ at $37^{\circ} \mathrm{C}$. The protein bands on the membrane were visualized using ECL chemiluminescence substrate (PE0010, Solarbio) and the protein levels were quantified with Gel-Pro-Analyzer software.

\section{Immunofluorescence}

Stomach and duodenum tissues were cut into $6 \mu \mathrm{m}$ thickness. Sections were incubated at $4^{\circ} \mathrm{C}$ overnight with the following primary antibodies: ghrelin (1:200, \#31,865, CST, Boston, USA), occludin (1:200, 66,378-1-Ig, Proteintech), and cyclooxygenase-2 (COX-2, 1:200, A1253, ABclonal, Wuhan, China). After three 5-min washing, the sections were cultivated with $\mathrm{Cy} 3$-conjugated goat anti-rabbit or anti-mouse antibody (1:400, A0516/A0521, Beyotime) at room temperature for $1 \mathrm{~h}$. The slides were stained with 4',6-diamidino-2-phenylindole (DAPI, C1002, Beyotime) and immunofluorescence was observed using light microscopy (BX53, Olympus, Tokyo, Japan) at original magnification $\times 600$.

\section{Statistical Analysis}

All data were shown as mean $\pm \mathrm{SD}$. Student's $t$-test (two tailed) and one-way analysis of variance (ANOVA) followed by Tukey's post hoc test were used to assess the difference among the groups. $\mathrm{P}<0.05$ was defined as statistically significant. GraphPad Prism 8.0 was used for statistical analysis of all data.

\section{Results}

\section{Paeoniflorin Promotes Gastric Emptying, Gastric Accommodation and Gastric Sensitivity in FD Rats}

The effect of paeoniflorin on IA-induced or CD-induced delayed gastric emptying was investigated by the phenol red method. It could be seen from Figure 1A, the gastric emptying of the control group administered IA or CD revealed an obvious decrease compared with the sham group $(\mathrm{p}<0.5)$. Paeoniflorin at a dose of $30 \mathrm{mg} / \mathrm{kg}$ markedly enhanced gastric emptying compared with the control group $(p<0.5)$. We also assessed the effect of paeoniflorin on gastric accommodation and gastric sensitivity. It could be seen from Figure 1B that enhanced balloon pressure of the sham group led to an increase in balloon volume. Compared with the sham group, balloon volume of the control rats induced by IA or CD was significantly decreased under distention pressures of $40 \mathrm{mmHg}, 60 \mathrm{mmHg}$ and 80 $\mathrm{mmHg}(\mathrm{p}<0.5)$, but not at $20 \mathrm{mmHg}$. Paeoniflorin treatment markedly improved gastric accommodation in FD rats $(p<0.5)$. On the other hand, EMS testing results displayed that FD rats of the control group extremely increased in EMG at distension pressures of $40 \mathrm{mmHg}, 60 \mathrm{mmHg}$ and $80 \mathrm{mmHg}$ compared with the sham rats $(\mathrm{p}<0.5$, Figure $1 \mathrm{C})$. Administration of paeoniflorin (15 and $30 \mathrm{mg} / \mathrm{kg} / \mathrm{daily}$ ) greatly repressed the EMG activity of FD rats to gastric distension $(\mathrm{p}<0.5)$. Our results suggested that paeoniflorin greatly ameliorated delayed gastric emptying, impaired gastric accommodation and gastric sensitivity in FD rats.

\section{Paeoniflorin Stimulates the Release of ACh in FD Rats}

To evaluate whether paeoniflorin stimulated the release of the neurotransmitter $\mathrm{ACh}$, we examined the $\mathrm{AChE}$ activity in paeoniflorin-treated FD rats. Figure $2 \mathrm{~A}$ and $\mathrm{B}$ showed that stomach-derived and hypothalamus-derived $\mathrm{AChE}$ activities of the control group were significantly increased by $C D$ administration in comparison to the sham group $(\mathrm{p}<0.5)$, but the effect induced by IA did not reach statistically significant. Moreover, paeoniflorin at doses of $15 \mathrm{mg} / \mathrm{kg}$ and $30 \mathrm{mg} / \mathrm{kg}$ efficiently suppressed AChE activity in stomach and hypothalamus of CD-induced rats $(p<0.5)$. The serum level of ACh in the control group was greatly downregulated by IA or CD administration compared with the sham group, but the use of paeoniflorin restored ACh level $(p<0.5$, Figure $2 C)$. Our results suggested that paeoniflorin treatment stimulated the release of $\mathrm{ACh}$ in FD rats.

\section{Paeoniflorin Regulates the Levels of Ghrelin and COX-2 in the Stomach of FD Rats}

Ghrelin was expressed as granular neuropeptides in the stratum basale of the stomach, as revealed by immunofluorescence (Figure 3A). Compared with the sham group, the expression of ghrelin was decreased in the stomach of FD rats $(\mathrm{p}<0.5$, Figure $3 \mathrm{~A}$ and $\mathrm{C})$. Paeoniflorin treatment enhanced the ghrelin expression of FD rats. Furthermore, the expression of COX-2 was observed in the stratum basale of the stomach in comparison to the sham group (Figure 
A

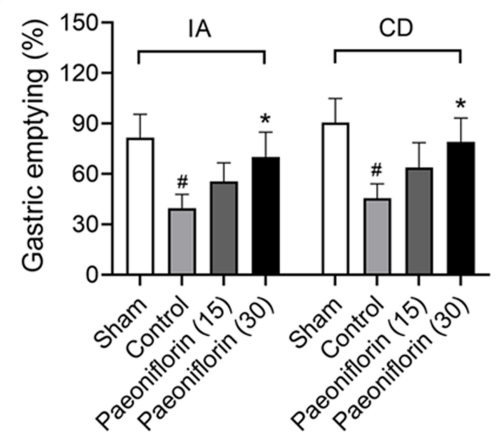

C

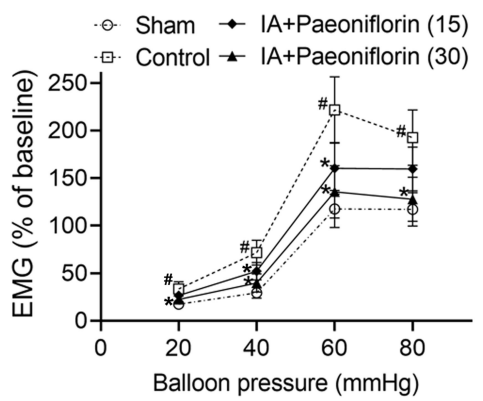

B
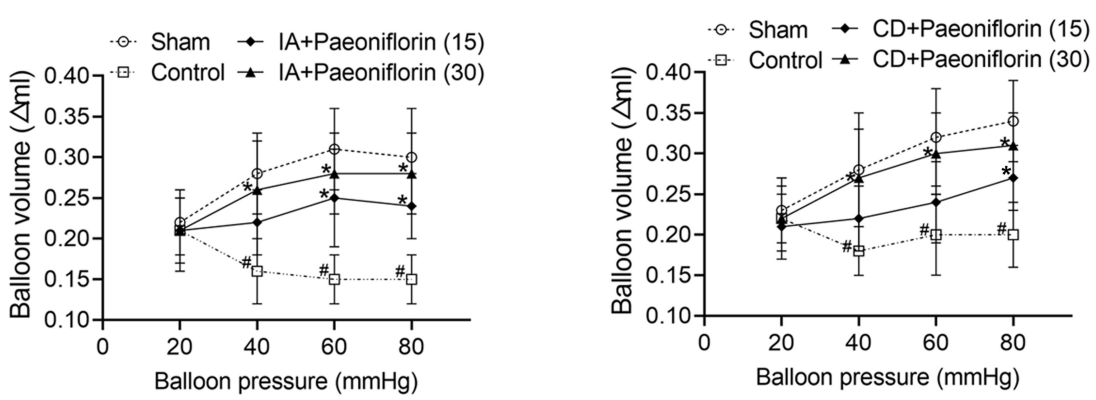

Figure I Paeoniflorin promotes gastric emptying, gastric accommodation and gastric sensitivity in FD rats. Rats were fasted for $18 \mathrm{~h}$ and then orally administered $0.2 \mathrm{~mL}$ of $0.1 \%$ iodoacetamide (IA) or subcutaneously administered clonidine $(C D)$ at a dose of $100 \mu \mathrm{g} / \mathrm{kg}$. Effects of 15 or $30 \mathrm{mg} / \mathrm{kg}$ of paeoniflorin on $(\mathbf{A})$ delayed gastric emptying, (B) gastric accommodation, and (C) gastric sensibility were assessed ( $n=6 /$ group). Values are mean \pm SD. ${ }^{\#}<0.05$ vs sham group; * $<0.05$ vs control group.

Abbreviation: EMG, electromyography

3B). However, the administration of paeoniflorin exhibited a reduction of COX-2 level in stomach of FD rats and thereby alleviating micro-inflammation $(p<0.5$, Figure $3 \mathrm{~B}$ and $\mathrm{D})$.

\section{Paeoniflorin Ameliorates the Impaired Duodenal Barrier in FD Rats}

A previous study has shown that duodenal barrier function is compromised in FD. ${ }^{26}$ Thus, we assessed whether adhesion proteins were altered in IA or CD-induced FD rats, and determined the effect of paeoniflorin on the expression of cell-to-cell adhesion proteins. The tight junction proteins (occludin and $\mathrm{ZO}-1$ ) and the adherens junction proteins (E-cadherin) were detected by Western blotting and immunofluorescence. It could be seen from Figure 4A that the expression of occludin and ZO-1 at the tight junction in the control group was greatly decreased in FD rats than those in the sham group $(\mathrm{p}<0.5)$. Paeoniflorin at a dose of $30 \mathrm{mg} / \mathrm{kg}$ obviously up-regulated occludin and ZO-1 levels in the duodenum of FD rats $(\mathrm{p}<0.5$, Figure 4A). In addition, no difference in protein expression of E-cadherin was observed in FD rats compared with the sham group. Paeoniflorin treatment had no effect on the adherens junction protein E-cadherin in the duodenum of FD rats (Figure 4A). The results of immunofluorescence revealed the reduced staining of occludin at the tight junction in control rats, and treatment with paeoniflorin effectively increased the occludin level (Figure 4B). Our results suggested that paeoniflorin ameliorated the impaired duodenal barrier in FD rats.

\section{Discussion}

Paeoniflorin, a glucoside, is one of the principal bioactive components of the P. lactiflora Pall and has many pharmacological functions, such as anti-inflammatory, antiallergic and antioxidant effects. ${ }^{15}$ Previous studies have found that paeoniflorin inhibits the AChE activity and promotes the 5-HT expression. ${ }^{19,21}$ AChE antagonist and 5- $\mathrm{HT}_{4}$ receptor agonists are commonly used in the treatment of FD due to the improvement of gastrointestinal motility. Therefore, this study examined the role and mechanism of paeoniflorin in rats with FD-like phenotype. Our results showed that paeoniflorin treatment accelerated gastric emptying and enhanced gastric accommodation as well as gastric sensitivity in IA or CD-induced FD rat models. Paeoniflorin stimulated the release of $\mathrm{ACh}$ in FD rats, 
A

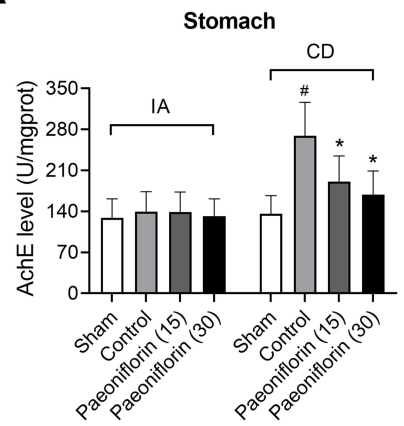

B

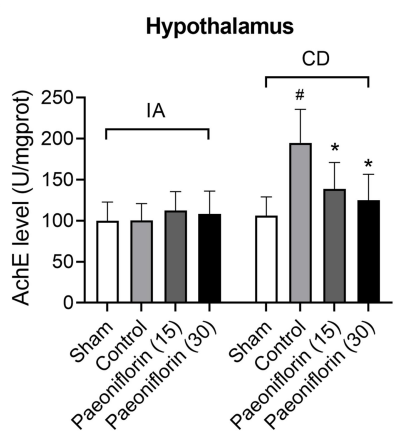

C

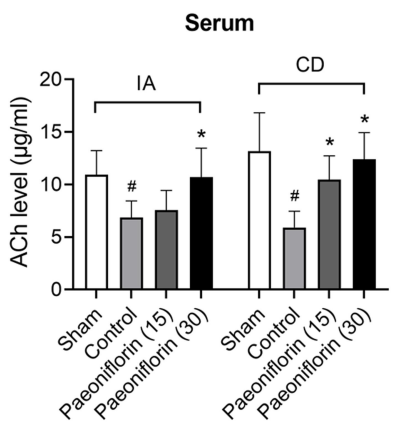

Figure 2 Paeoniflorin stimulates the release of acetylcholine (ACh) in FD rats. (A) Stomach-derived and (B) hypothalamus-derived acetylcholinesterase (AchE) activities were measured by paeoniflorin administration at dose of 15 or $30 \mathrm{mg} / \mathrm{kg}(\mathrm{n}=6 / \mathrm{group})$. (C) The serum level of acetylcholine (ACh) was investigated $(\mathrm{n}=6 / \mathrm{group})$. Values are mean \pm SD. ${ }^{\#} \mathrm{P}<0.05$ vs sham group; ${ }^{*} \mathrm{p}<0.05$ vs control group.

accompanied with increased ghrelin and reduced COX-2. Besides, paeoniflorin administration ameliorated the impaired duodenal barrier in FD rats. These findings indicate the therapeutic effect of paeoniflorin in FD rats.

Adverse events in early life are one of risk factors for developing FD in adulthood. ${ }^{27}$ Using a previously described model of FD rats where 10-day-old rat pups got gastric irritation within a few days, we find that IA treatment results in the delayed gastric emptying, impaired gastric accommodation and visceral hypersensitivity in adult rats, which is in conformity to a previous study. ${ }^{22}$ The administration of paeoniflorin restores gastric motility in IAinduced adult rats. Besides, a model of adult rats with $\mathrm{CD}$ treatment is established to induce FD-like phenotype. CD, a $\alpha 2$-adrenoceptor agonist, induces gastrointestinal dysfunction through suppressing the release of ACh from cholinergic nerve terminals. Gastrointestinal dysfunction models induced by $\alpha 2$-adrenoceptor agonists have been widely applied to assess the influence of gastroprokinetic drugs in animals. ${ }^{28}$ Sninsky et al have confirmed that gastrointestinal dysfunction is induced by an $\alpha 2$-adrenoceptor agonist in humans. ${ }^{29}$ We find that $\mathrm{CD}$ treatment leads to delayed gastric emptying, impaired gastric accommodation and visceral hypersensitivity in adult rats. These results with $\mathrm{CD}$ treatment are in line with a previous study. ${ }^{30}$ Similarly, administration of paeoniflorin alleviates delayed gastric emptying, impaired gastric accommodation and sensitivity in a concentration-dependent manner. Therefore, we determine that paeoniflorin can efficiently ameliorate the gastrointestinal dysfunction induced by a different cause.

As we all know, the central nervous system affects gastrointestinal function. In the central nervous system, vagal efferent nerve fibers project to the stomach, which play a vital role in gastric motility, and thereby stimulating
ACh release from cholinergic nerve terminals in the myenteric plexus of the stomach. ${ }^{31}$ A study has reported that itopride promotes $\mathrm{ACh}$ release from cholinergic nerves through antagonizing dopamine D2 receptors with antiAChE activity. ${ }^{32}$ Another study has also found that mosapride induces $\mathrm{ACh}$ release from cholinergic nerves and facilitates gastric motility via the stimulation of $5-\mathrm{HT}_{4}$ receptors. ${ }^{33}$ Consequently, the release of $\mathrm{ACh}$ at the cholinergic receptor sites plays an important in gastric motility. In the present study, we find that treatment with $\mathrm{CD}$ significantly increases the rat stomach and hypothalamusderived AChE activities, but a weak effect is observed on AChE activity in IA-induced rats. Administration of paeoniflorin stimulates the release of Ach in IA or CD-induced FD rats. Therefore, we determine that paeoniflorin contributes to gastric motility and the release of ACh by inhibiting $\mathrm{AChE}$ activity in Cd-induced FD rats. Gastric irritation with IA in the neonatal period induces superficial sloughing of the gastric epithelium and mild gastritis, consequently leading to impaired gastric sensory and motor function. ${ }^{20}$ The therapeutic effect of paeoniflorin in IAinduced FD rats may be due to paeoniflorin up-regulates the cAMP and 5-HT expression, thereby promoting the release of ACh. ${ }^{19,34,35}$ Our researches determine that paeoniflorin contributes to the release of $\mathrm{ACh}$, and further relieves abnormalities in rats with FD.

Treatment with IA leads to the production of ghrelin in the stomach, and then ghrelin is released into the circulation. ${ }^{36}$ Ghrelin can directly affect the activity of the arcuate nucleus neurons. The hypothalamus modulates energy expenditure after receiving both hormonal signals and peripheral neurons. ${ }^{37}$ Therefore, the release of circulating hormones, such as ghrelin, modulates the brain-gut axis through hormonal signals. ${ }^{16}$ Ghrelin has 
A

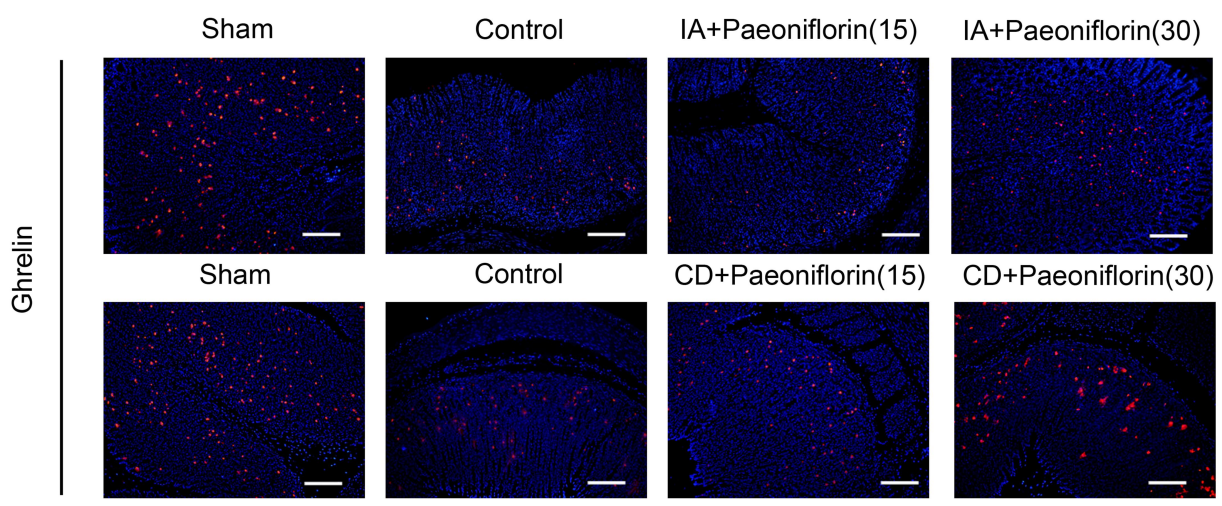

B

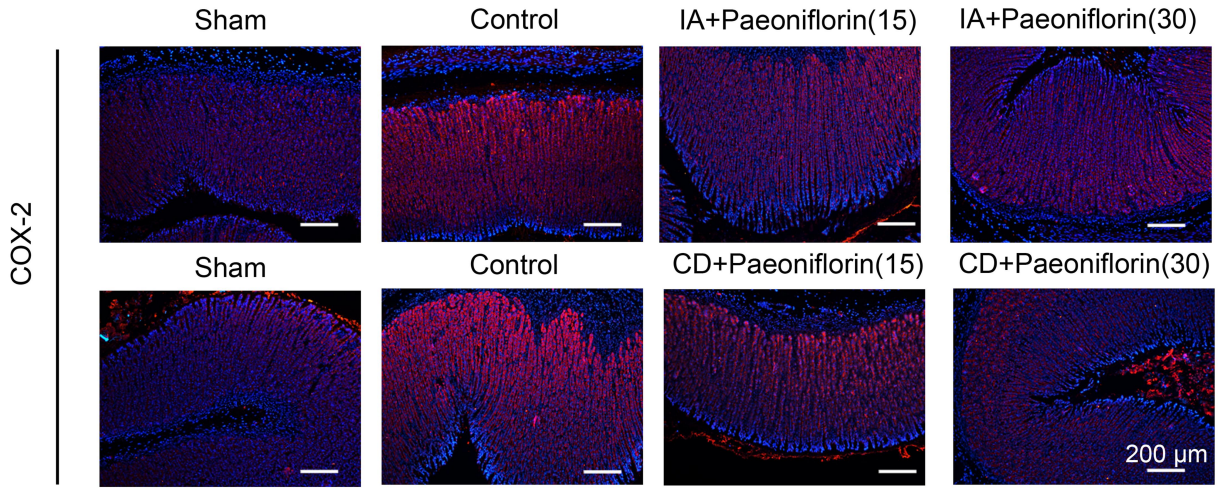

C
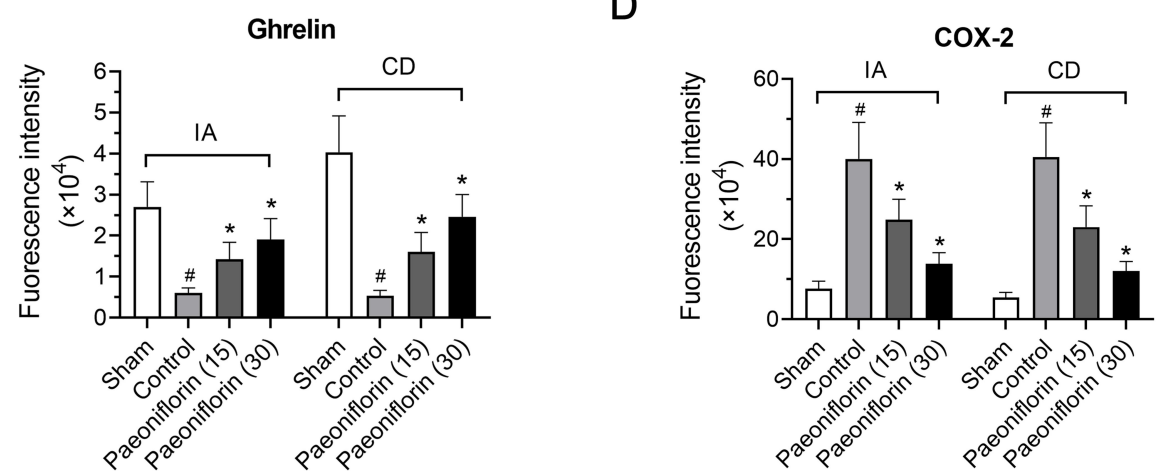

Figure 3 Paeoniflorin regulates the levels of ghrelin and cyclo-oxygenase-2 (COX-2) in the stomach of FD rats. (A) Immunofluorescence images for ghrelin in the stomach were shown and $(\mathbf{C})$ corresponding immunofluorescence intensity was quantified $(n=6 /$ group). (B) Immunofluorescence images for COX-2 in the stomach was shown and (D) corresponding immunofluorescence intensity was quantified $(n=6 /$ group). Red cells were positive for ghrelin and COX-2. Bars $=200 \mu \mathrm{m}$.

been reported to efficiently increase gastric peristalsis. ${ }^{25}$ Ghrelin levels are markedly lower in patients with FD compared to that in healthy volunteers. ${ }^{38}$ A previous study has shown the reduced expression of ghrelin in the brain and stomach of FD rats induced by IA. ${ }^{39}$ Consistent with this finding, our study finds that gastric irritation in the neonatal rat can lead to a reduction of ghrelin compared with the sham rats. Moreover, we are the first to confirm that the expression of ghrelin is reduced in the stomach of FD rats induced by $\mathrm{CD}$. The administration of paeoniflorin markedly enhanced ghrelin expression, especially at a dose of $30 \mathrm{mg} / \mathrm{kg}$. In addition, it has been shown that micro-inflammation exists in gastrointestine of FD patients. ${ }^{40} \mathrm{COX}-2$ is the inducible enzyme that is less expressed in normal tissues but highly expressed when stimulated by inflammation. ${ }^{41} \mathrm{~A}$ previous study found that COX-2 was highly expressed in the stomach of FD rats. ${ }^{42}$ Besides, COX-2 inhibitors significantly reduced the risk of gastrointestinal adverse effects in patients. ${ }^{43}$ Another study has shown the protective effects of paeoniflorin on rheumatoid arthritis by reducing the COX-2 
A

ZO-1 - - $-230 \mathrm{kDa}$ E-cadherin - - $125 \mathrm{kDa}$ Occludin - - $59 \mathrm{kDa}$

$\beta$-actin $-20-42 \mathrm{kDa}$

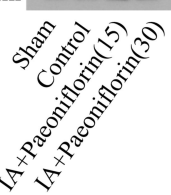

B
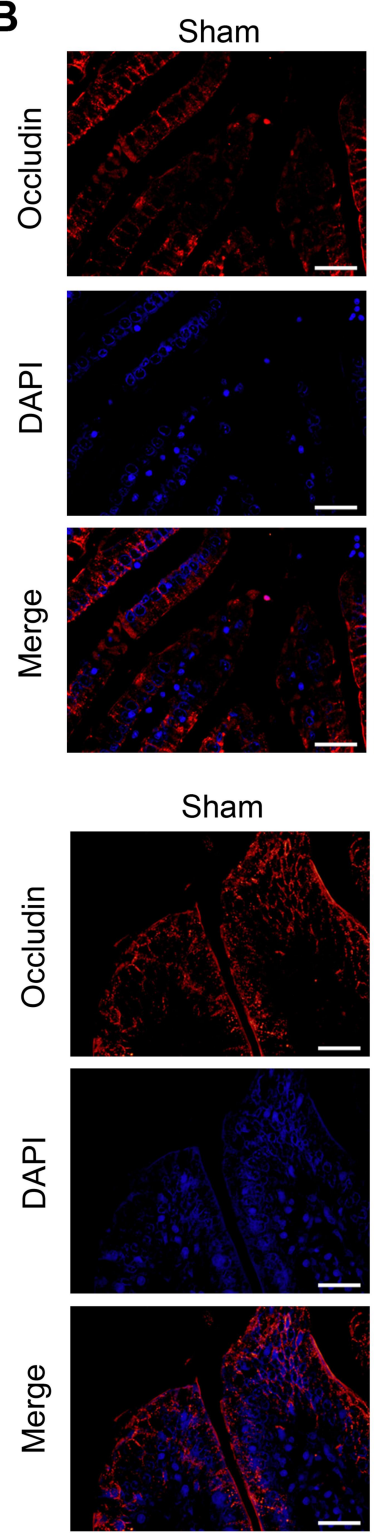

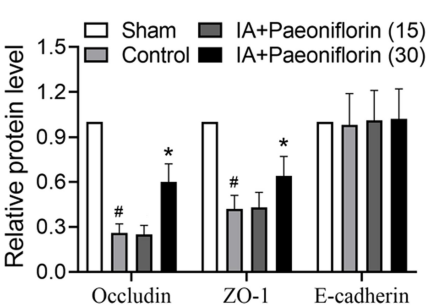

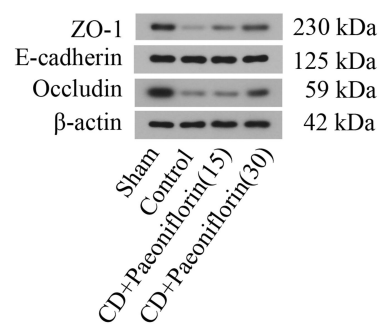

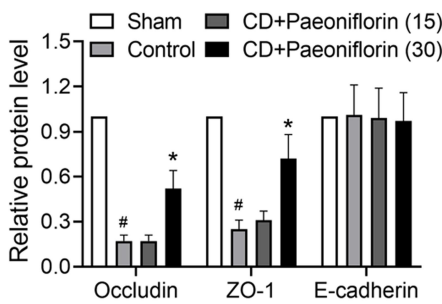

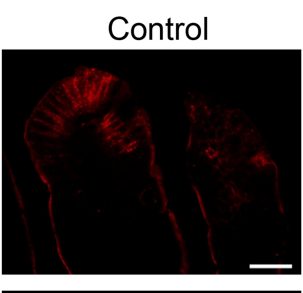
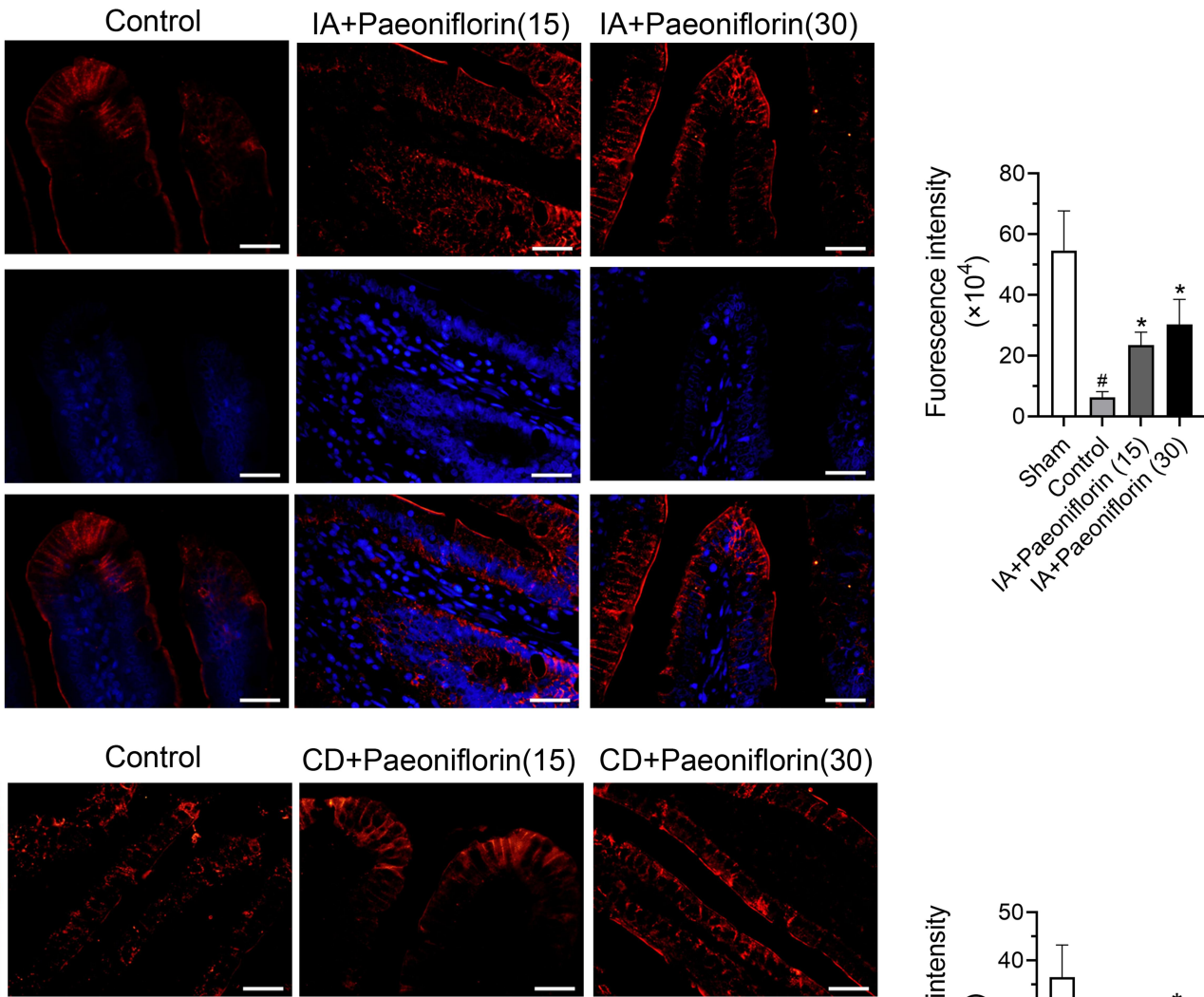

CD+Paeoniflorin(15)
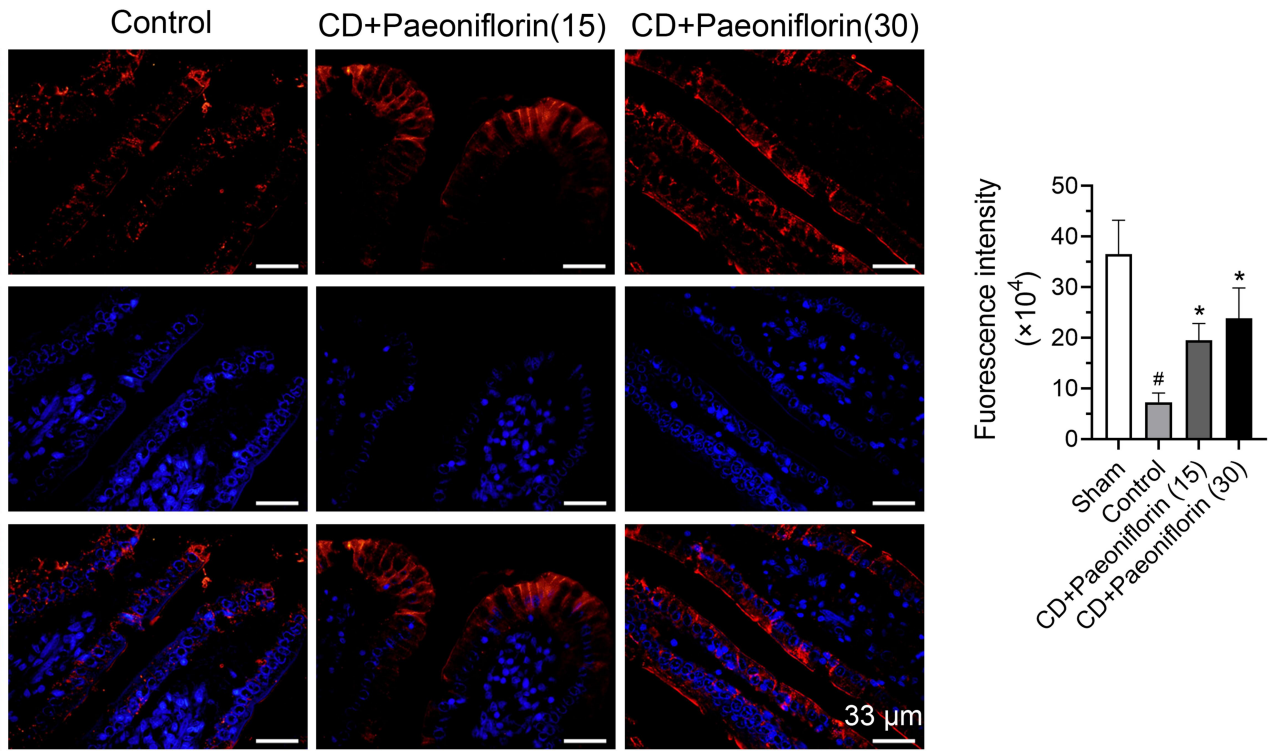

Figure 4 Paeoniflorin ameliorates the impaired gastrointestinal mucosa in FD rats. (A) Effects of paeoniflorin on adhesion proteins, including occluding, Zona Occludens-I (ZO-I) and E-cadherin, in the duodenal mucosa were assessed by Western blotting $(n=6 /$ group $)$. (B) Quantitative immunofluorescence analysis was used to detect the expression of occludin. Bars $=33 \mu \mathrm{m}$. Values are mean $\pm \mathrm{SD}$. ${ }_{\mathrm{P}} \mathrm{P}<0.05$ vs sham group; ${ }_{\mathrm{p}}<0.05$ vs control group.

protein expression level. ${ }^{44}$ In agreement with this report, our study determines that paeoniflorin treatment efficiently down-regulates the level of COX-2 and thereby alleviates abnormalities in rats with $\mathrm{FD}$.
The intestinal barrier plays an important in preventing the translocation of harmful substances from gut lumen to submucosa. Previous study has confirmed that duodenal barrier is implicated in the pathomechanism of $\mathrm{FD}^{45}$ 
Adhesion proteins are significant components of the intestinal barrier, including the tight junction proteins (occludin and ZO-1) and the adherens junction proteins (E-cadherin). ${ }^{46}$ The dysregulation of adhesion proteins associates with impaired duodenal integrity and microinflammation. Downregulation of occludin leads to an increase of permeability, thereby triggering intestinal diseases. ${ }^{47}$ A study finds that tail clamping-associated stress leads to a decrease in occludin expression in the duodenum, thereby damaging duodenal integrity of FD rats. ${ }^{48}$ Another study has also reported the impaired duodenal mucosal barrier function in patients with FD, exhibited by a reduction of ZO-1 and E-cadherin levels. ${ }^{26}$ In the present study, the decrease in occludin and ZO-1 expression in the duodenum of FD rats is induced by IA or CDassociated stress. Administration of paeoniflorin causes the upregulation of tight junction proteins (occludin and $\mathrm{ZO}$ 1) in the duodenum. Therefore, we postulate that paeoniflorin up-regulates the expression of occludin and ZO-1 expression in the duodenum by changes of neurotransmitter $\mathrm{ACh}$ in FD rats.

\section{Conclusion}

In summary, paeoniflorin possesses the ability to relieve the symptoms of FD, with attenuated inflammatory reaction. Paeoniflorin inhibits the AChE activity and increases the levels of $\mathrm{ACh}$ and ghrelin. Furthermore, paeoniflorin ameliorates the impaired duodenal barrier by up-regulating the expression of adhesion proteins (occludin and ZO-1). These findings suggest that paeoniflorin is a potential therapeutic compound for FD.

\section{Acknowledgments}

This study was supported by grants from the National Natural Science Foundation of China [Nos. 81673727 and 81273919].

\section{Disclosure}

The authors report no conflicts of interest in this work.

\section{References}

1. Talley NJ, Locke GR 3rd, Lahr BD, et al. Functional dyspepsia, delayed gastric emptying, and impaired quality of life. Gut. 2006;55 (7):933-939. doi:10.1136/gut.2005.078634

2. Curran MP, Robinson DM. Mosapride in gastrointestinal disorders. Drugs. 2008;68(7):981-991. doi:10.2165/00003495-200868070-00007

3. El-Serag HB, Talley NJ. Systemic review: the prevalence and clinical course of functional dyspepsia. Aliment Pharmacol Ther. 2004;19 (6):643-654. doi:10.1111/j.1365-2036.2004.01897.x
4. Lacy BE, Weiser KT, Kennedy AT, Crowell MD, Talley NJ. Functional dyspepsia: the economic impact to patients. Aliment Pharmacol Ther. 2013;38(2):170-177. doi:10.1111/apt.12355

5. Enck P, Azpiroz F, Boeckxstaens G, et al. Functional dyspepsia. Nat Rev Dis Primers. 2017;3:17081.

6. Asano H, Tomita T, Nakamura K, et al. Prevalence of gastric motility disorders in patients with functional dyspepsia. J Neurogastroenterol Motil. 2017;23(3):392-399. doi:10.5056/jnm16173

7. Tonini M, Cipollina L, Poluzzi E, Crema F, Corazza GR, De Ponti F. Review article: clinical implications of enteric and central D2 receptor blockade by antidopaminergic gastrointestinal prokinetics. Aliment Pharmacol Ther. 2004;19(4):379-390. doi:10.1111/j.13652036.2004.01867.x

8. O'Mahony S, Dinan TG, Keeling PW, Chua AS. Central serotonergic and noradrenergic receptors in functional dyspepsia. World J Gastroenterol. 2006;12(17):2681-2687. doi:10.3748/wjg.v12.117.2681

9. Holtmann G, Gapasin J. Failed therapy and directions for the future in dyspepsia. Dig Dis. 2008;26(3):218-224. doi:10.1159/000121350

10. Rao AS, Camilleri M. Review article: metoclopramide and tardive dyskinesia. Aliment Pharmacol Ther. 2010;31(1):11-19. doi:10.1111/ j.1365-2036.2009.04189.x

11. Doggrell SA, Hancox JC. Cardiac safety concerns for domperidone, an antiemetic and prokinetic, and galactagogue medicine. Expert Opin Drug Saf. 2014;13(1):131-138. doi:10.1517/ 14740338.2014.851193

12. Tack J, Talley NJ, Camilleri M, et al. Functional gastroduodenal disorders. Gastroenterology. 2006;130(5):1466-1479. doi:10.1053/j. gastro.2005.11.059

13. Monkemuller K, Malfertheiner P. Drug treatment of functional dyspepsia. World J Gastroenterol. 2006;12(17):2694-2700. doi:10.3748/wjg.v12.i17.2694

14. Chen LC, Lee MH, Chou MH, Lin MF, Yang LL. Pharmacokinetic study of paeoniflorin in mice after oral administration of Paeoniae radix extract. J Chromatogr B Biomed Sci Appl. 1999;735(1):33-40. doi:10.1016/S0378-4347(99)00408-9

15. Zhong SZ, Ge QH, Li Q, Qu R, Ma SP. Peoniflorin attentuates Abeta $((1-42))$-mediated neurotoxicity by regulating calcium homeostasis and ameliorating oxidative stress in hippocampus of rats. J Neurol Sci. 2009;280(1-2):71-78. doi:10.1016/j.jns.2009.01.027

16. Liu HQ, Zhang WY, Luo XT, Ye Y, Zhu XZ. Paeoniflorin attenuates neuroinflammation and dopaminergic neurodegeneration in the MPTP model of Parkinson's disease by activation of adenosine A1 receptor. $B r J$ Pharmacol. 2006;148(3):314-325. doi:10.1038/sj. bjp.0706732

17. Mao QQ, Zhong XM, Qiu FM, Li ZY, Huang Z. Protective effects of paeoniflorin against corticosterone-induced neurotoxicity in PC12 cells. Phytother Res. 2012;26(7):969-973. doi:10.1002/ptr.3673

18. Lan Z, Chen L, Fu Q, et al. Paeoniflorin attenuates amyloid-beta peptide-induced neurotoxicity by ameliorating oxidative stress and regulating the NGF-mediated signaling in rats. Brain Res. 2013;1498:9-19. doi:10.1016/j.brainres.2012.12.040

19. Qiu ZK, He JL, Liu X, et al. Anxiolytic-like effects of paeoniflorin in an animal model of post traumatic stress disorder. Metab Brain Dis. 2018;33(4):1175-1185. doi:10.1007/s11011-018-0216-4

20. Liu LS, Winston JH, Shenoy MM, Song GQ, Chen JD, Pasricha PJ. A rat model of chronic gastric sensorimotor dysfunction resulting from transient neonatal gastric irritation. Gastroenterology. 2008;134 (7):2070-2079. doi:10.1053/j.gastro.2008.02.093

21. Asano T, Aida S, Suemasu S, Tahara K, Tanaka K, Mizushima T. Aldioxa improves delayed gastric emptying and impaired gastric compliance, pathophysiologic mechanisms of functional dyspepsia. Sci Rep. 2015;5:17519. doi:10.1038/srep17519

22. Hagiwara SI, Kaushal E, Paruthiyil S, Pasricha PJ, Hasdemir B, Bhargava A. Gastric corticotropin-releasing factor influences mast cell infiltration in a rat model of functional dyspepsia. PLoS One. 2018;13(9):e0203704. doi:10.1371/journal.pone.0203704 
23. Kawachi M, Matsunaga Y, Tanaka T, et al. Acotiamide hydrochloride (Z-338) enhances gastric motility and emptying by inhibiting acetylcholinesterase activity in rats. Eur $J$ Pharmacol. 2011;666(1-3):218-225. doi:10.1016/j.ejphar.2011.05.049

24. Uchida M, Kobayashi O, Iwamoto C. Effects of L-tryptophan on gastric emptying evaluated by breath test in relation to gastric accommodation evaluated by Barostat in rats. J Pharmacol Sci. 2015;127 (2):229-231. doi:10.1016/j.jphs.2014.12.008

25. Liu J, Li F, Tang XD, et al. XiangshaLiujunzi decoction alleviates the symptoms of functional dyspepsia by regulating brain-gut axis and production of neuropeptides. BMC Complement Altern Med. 2015;15:387. doi:10.1186/s12906-015-0913-z

26. Vanheel H, Vicario M, Vanuytsel T, et al. Impaired duodenal mucosal integrity and low-grade inflammation in functional dyspepsia. Gut. 2014;63(2):262-271. doi:10.1136/gutjnl-2012-303857

27. Liu S, Hagiwara SI, Bhargava A. Early-life adversity, epigenetics, and visceral hypersensitivity. Neurogastroenterol Motil. 2017;29:9. doi:10.1111/nmo.13170

28. Ueki S, Matsunaga Y, Yoneta T, Tamaki H, Itoh Z. Gastroprokinetic activity of nizatidine during the digestive state in the dog and rat. Arzneimittelforschung. 1999;49(7):618-625.

29. Sninsky CA, Davis RH, Clench MH, Thomas KD, Mathias JR. Effect of lidamidine hydrochloride and loperamide on gastric emptying and transit of the small intestine. A double-blind study. Gastroenterology. 1986;90(1):68-73. doi:10.1016/0016-5085(86)90076-4

30. Fulop K, Zadori Z, Ronai AZ, Gyires K. Characterisation of alpha2-adrenoceptor subtypes involved in gastric emptying, gastric motility and gastric mucosal defence. Eur J Pharmacol. 2005;528 (1-3):150-157. doi:10.1016/j.ejphar.2005.10.025

31. Kawachi M, Hori N, Takei M, Kurimoto T, Akaike N, Ito Y. Gastric relaxation induced by electrical and chemical stimulation of the area postrema in the rat. Gen Physiol Biophys. 2008;27(4):243-252.

32. Iwanaga $\mathrm{Y}$, Miyashita N, Morikawa K, Mizumoto A, Kondo Y, Itoh Z. A novel water-soluble dopamine-2 antagonist with anticholinesterase activity in gastrointestinal motor activity. Comparison with domperidone and neostigmine. Gastroenterology. 1990;99 (2):401-408. doi:10.1016/0016-5085(90)91022-X

33. Mine Y, Yoshikawa T, Oku S, Nagai R, Yoshida N, Hosoki K. Comparison of effect of mosapride citrate and existing 5-HT4 receptor agonists on gastrointestinal motility in vivo and in vitro. J Pharmacol Exp Ther. 1997;283(3):1000-1008.

34. Chen JY, Wu HX, Chen Y, et al. Paeoniflorin inhibits proliferation of fibroblast-like synoviocytes through suppressing G-protein-coupled receptor kinase 2. Planta Med. 2012;78(7):665-671. doi:10.1055/ s-0031-1298327

35. Hatat B, Yahiaoui S, Lecoutey C, et al. A novel in vivo anti-amnesic agent, specially designed to express both acetylcholinesterase (AChE) inhibitory, serotonergic subtype 4 receptor (5-HT4R) agonist and serotonergic subtype 6 receptor (5-HT6R) inverse agonist activities, with a potential interest against alzheimer's disease. Front Aging Neurosci. 2019;11:148.

36. Tomita R. Regulation of vasoactive intestinal peptide and substance P in the human pyloric sphincter. Hepatogastroenterology. 2009;56 (94-95):1403-1406.

Drug Design, Development and Therapy

\section{Publish your work in this journal}

Drug Design, Development and Therapy is an international, peerreviewed open-access journal that spans the spectrum of drug design and development through to clinical applications. Clinical outcomes, patient safety, and programs for the development and effective, safe, and sustained use of medicines are a feature of the journal, which has also
37. Murphy KG, Dhillo WS, Bloom SR. Gut peptides in the regulation of food intake and energy homeostasis. Endocr Rev. 2006;27 (7):719-727. doi:10.1210/er.2006-0028

38. Yagi T, Asakawa A, Ueda H, Miyawaki S, Inui A. The role of ghrelin in patients with functional dyspepsia and its potential clinical relevance (Review). Int J Mol Med. 2013;32(3):523-531. doi:10.3892/ ijmm.2013.1418

39. Ganko M, Calka J. Prolonged acetylsalicylic-acid-supplementationinduced gastritis affects the chemical coding of the stomach innervating vagal efferent neurons in the porcine dorsal motor vagal nucleus (DMX). J Mol Neurosci. 2014;54(2):188-198. doi:10.1007/s12031014-0274-y

40. Du L, Chen B, Kim JJ, Chen X, Dai N. Micro-inflammation in functional dyspepsia: A systematic review and meta-analysis. Neurogastroenterol Motil. 2018;30(4):e13304. doi:10.1111/ nmo.13304

41. Zhang S, Bian H, Li X, et al. Hydrogen sulfide promotes cell proliferation of oral cancer through activation of the COX2/AKT/ ERK1/2 axis. Oncol Rep. 2016;35(5):2825-2832. doi:10.3892/ or.2016.4691

42. He Y, Yang C, Wang P, et al. Child compound Endothelium corneum attenuates gastrointestinal dysmotility through regulating the homeostasis of brain-gut-microbiota axis in functional dyspepsia rats. J Ethnopharmacol. 2019;240:111953. doi:10.1016/j.jep.2019.111953

43. Jarupongprapa S, Ussavasodhi P, Katchamart W. Comparison of gastrointestinal adverse effects between cyclooxygenase-2 inhibitors and non-selective, non-steroidal anti-inflammatory drugs plus proton pump inhibitors: a systematic review and meta-analysis. $J$ Gastroenterol. 2013;48(7):830-838. doi:10.1007/s00535-0120717-6

44. Jia Z, He J. Paeoniflorin ameliorates rheumatoid arthritis in rat models through oxidative stress, inflammation and cyclooxygenase 2. Exp Ther Med. 2016;11(2):655-659. doi:10.3892/etm.2015.2908

45. Tanaka F, Tominaga K, Fujikawa Y, et al. Concentration of glial cell line-derived neurotrophic factor positively correlates with symptoms in functional dyspepsia. Dig Dis Sci. 2016;61(12):3478-3485. doi:10.1007/s10620-016-4329-5

46. Van Itallie CM, Anderson JM. Architecture of tight junctions and principles of molecular composition. Semin Cell Dev Biol. 2014;36:157-165. doi:10.1016/j.semcdb.2014.08.011

47. Xiao Y, Liu YY, Yu KQ, Ouyang MZ, Luo R, Zhao XS. Chinese herbal medicine liu jun zi tang and xiang sha liu jun zi tang for functional dyspepsia: meta-analysis of randomized controlled trials. Evid Based Complement Alternat Med. 2012;2012:936459. doi:10.1155/2012/936459

48. Chang X, Zhao L, Wang J, Lu X, Zhang S. Sini-san improves duodenal tight junction integrity in a rat model of functional dyspepsia. BMC Complement Altern Med. 2017;17(1):432. doi:10.1186/s12906-017-1938-2

been accepted for indexing on PubMed Central. The manuscript management system is completely online and includes a very quick and fair peer-review system, which is all easy to use. Visit http://www. dovepress.com/testimonials.php to read real quotes from published authors. 